\title{
AVALIAÇÃO DA QUALIDADE DE VIDA NA POPULAÇÃO PORTUGUESA
}

\author{
Marilene Andrade Lopes \\ Enfermeira, Escola Superior de Saúde. Portugal \\ marileneanyviny@gmail.com \\ Jéssica Andreia Roças Parente \\ Enfermeira, Escola Superior de Saúde \\ Manuel Alberto Morais Brás \\ Instituto Politécnico de Bragança, Investigador Integrado no \\ CINTESIS e Professor na Escola Superior de Saúde de Bragança, Portugal \\ Maria Fátima Pereira Geraldes \\ Enfermeira, ULSNe. Portugal \\ Eugénia Maria Garcia Jorge Anes \\ Instituto Politécnico de Bragança, Investigadora Integrada da \\ UICISA: e professora na Escola de Saúde IPB. Bragança, Portugal
}

Recepción Artículo: 17 mayo 2021

Admisión Evaluación: 17 mayo 2021

Informe Evaluador 1: 19 mayo 2021

Informe Evaluador 2: 21 mayo 2021

Aprobación Publicación: 01 junio 2021

\section{RESUMO}

Nas últimas décadas a avaliação da qualidade de vida das populações tem sido constante, quer em termos gerais, quer em populações específicas, muitas vezes utilizadas para avaliar o impacto das intervenções de saúde. 0 seu conceito pode ser diverso mediante o contexto ou a diferente perceção dos sujeitos. A Organização Mundial de Saúde entende-a como a perceção que um indivíduo tem sobre a sua posição na vida, no seu contexto, atendendo aos seus objetivos, expetativas, padrões e preocupações. A saúde e a qualidade de vida são dois conceitos com uma relação incontestável, uma vez que "a saúde contribui para melhorar a qualidade de vida dos indivíduos e esta é determinante essencial para que os indivíduos ou a comunidade tenham saúde. Assim pretendemos avaliar a qualidade de vida nos indivíduos com idade igual ou superior a 18 anos. Para tal, optámos por realizar um estudo descritivo de cariz transversal com uma abordagem quantitativa. Foi usada uma metodologia denominada bola de neve ou snowball sampling, sendo uma técnica de amostragem não probabilística. Foi utilizado um questionário constituído por questões de caraterização e pelo SF-36v2 (The Medical Outcomes Study 36-item Short Form Health Survey). Este é um questionário genérico de saúde, com 36 itens que avaliam a dimensão física, emocional e social, assim como os desempenhos físico e emocional, a dor e a saúde geral e vitalidade. A colheita de dados foie efetuada em 2020. Foram tidos em conta os preceitos éticos aplicados a este tipo de estudos, tendo em conta a Declaração de Helsínquia e Convenção de Oviedo. A amostra é constituída por 135 indivíduos, maioritariamente do sexo feminino (68,15\%), com idades compreendidas entre os 18 e os 63 anos. São maioritaria- 


\section{AVALIAÇÃO DA QUALIDADE DE VIDA NA POPULAÇÃO PORTUGUESA}

mente solteiros (60,74\%), licenciados (50,37\%), residentes em zona urbana (70,37\%). Relativamente ao estado de saúde, percecionam-na boa e excelente (48,88\%), comparativamente a um ano atrás 45,93\% percecionam-na semelhante. Em termos físicos, sentem quase sempre vitalidade 37,5\% dos questionados e algumas vezes $27,94 \%$. Em termos psicológicos e emocionais $43,38 \%$ sentem tranquilidade e calma a maior parte do tempo e 23,53 sentem algum tempo. Mais de 50\% não sentem qualquer limitação nas atividades quer física quer psicológica ou emocional. Sentem-se tão saudáveis ou mais como os outros $62,24 \%$. Estes resultados permitem-nos concluir que em geral os participantes apresentam bons índices de qualidade de vida relacionada. Resultados semelhantes foram encontrados no que respeita à saúde física e saúde emocional. 0 tamanho da amostra pode ser considerado como limite desta investigação. Estas avaliações permitem a identificação dos determinantes da saúde e facilitam aos a tomada de decisão.

Palavras-chave: qualidade de vida; SF-36

\section{ABSTRACT}

Quality of life assessment in the portuguese population. In the last decades, the evaluation of the quality of life of populations has been constant, both in general terms and in specific populations, often used to assess the impact of health interventions. Its concept can be different due to the context or the different perception of the subjects. The World Health Organization understands it as the perception that an individual has about his position in life, in his context, meeting his goals, expectations, standards and concerns. Health and quality of life are two concepts with an indisputable relationship, since "health contributes to improving the quality of life of individuals and this is an essential determinant for individuals or the community to have health. Thus, we intend to evaluate the quality of life in individuals aged 18 years or over. To this end, we opted to carry out a descriptive cross-sectional study with a quantitative approach. A methodology called snowball or snowball sampling was used, being a non-probabilistic sampling technique. A questionnaire consisting of characterization questions and the SF-36v2 (The Medical Outcomes Study 36-item Short Form Health Survey) was used. This is a generic health questionnaire, with 36 items that assess the physical, emotional and social dimension, as well as physical and emotional performance, pain and general health and vitality. Data collection was carried out in 2020. The ethical precepts applied to this type of studies were considered, taking into account the Declaration of Helsinki and the Oviedo Convention. The sample consists of 135 individuals, mostly female (68.15\%), aged between 18 and 63 years. They are mostly single (60.74\%), licensed (50.37\%), residing in urban areas $(70.37 \%)$. Regarding the state of health, they perceive it to be good and excellent (48.88\%), compared to a year ago $45.93 \%$ perceive it to be similar. In physical terms, $37.5 \%$ of those questioned and sometimes $27.94 \%$ feel vitality. In psychological and emotional terms $43.38 \%$ feel calm and calm most of the time and 23.53 feel some time. More than $50 \%$ do not feel any limitation in activities, whether physical, psychological, or emotional. They feel as healthy or more like the other $62.24 \%$. These results allow us to conclude that in general the participants have good levels of related quality of life. Similar results were found about physical and emotional health. The sample size can be considered as a limit for this investigation. These assessments allow the identification of health determinants and facilitate decision making.

Keywords: Quality of life; SF-36

\section{INTRODUÇÃO}

Nas últimas décadas a avaliação da qualidade de vida das populações tem sido constante, quer em termos gerais, quer em populações específicas, muitas vezes utilizadas para avaliar o impacto das intervenções de saúde. 0 seu conceito pode ser diverso mediante 0 contexto ou a diferente perceção dos sujeitos.

A Organização Mundial de Saúde entende-a como a perceção que um indivíduo tem sobre a sua posição na vida, no seu contexto, atendendo aos seus objetivos, expetativas, padrões e preocupações. A saúde e a qualidade de vida são dois conceitos com uma relação incontestável, uma vez que "a saúde contribui para melhorar a qualidade de vida dos indivíduos e esta é determinante essencial para que os indivíduos ou a comunidade tenham saúde. 
Sendo um fator essencial da vida humana, a saúde propicia ao indivíduo um estado de bem-estar, o que the garante um desemprenho satisfatório nos diversos planos: a nível físico, psicológico e social (Nunes \& Rego, 2002).

No domínio da saúde, existem diversos aspetos da vida do indivíduo que se podem configurar como determinantes da própria saúde, desde os aspetos cotados mais negativamente (por exemplo a morte) até aos aspetos mais positivos da vida (Guyatt, Feeny, \& Patrick, 1993).

Os comportamentos da pessoa são influenciados pelo ambiente no qual ela vive e se desenvolve. 0 ambiente no qual as pessoas vivem e se desenvolvem é constituído por elementos humanos, físicos, políticos, económicos, culturais e organizacionais, que condicionam e influenciam os estilos de vida e que se repercutem no conceito de saúde. Na prática dos cuidados, os enfermeiros necessitam de focalizar a sua intervenção na complexa interdependência pessoa / ambiente (OE, 2011).

Em 2005, a OMS criou a Comissão sobre Determinantes Sociais da Saúde (Commission on Social Determinants of Health - CSDH), com o objetivo de "promover, em âmbito internacional, uma tomada de consciência sobre a importância dos determinantes sociais na situação de saúde de indivíduos e populações e sobre a necessidade do combate às iniquidades em saúde por eles geradas" (Melo, 2020).

Na literatura podemos encontrar várias definições de qualidade de vida. A primeira alusão a este conceito foi feita em 1964 pelo político Lyndon Johnson, que afirmou que "os objetivos da economia não podem ser medidos através do balanço dos bancos. Eles só podem ser medidos através da qualidade de vida que proporcionam às pessoas" (Fleck, Leal, Louzada, Xavier, Chachamovich, Vieira, Santos, \& Pinzon, 1999).

Na década de 70 foi publicado o relatório da Commission on National Goals, que deixou subjacente a preocupação com o bem-estar e a qualidade de vida das pessoas no período pós-guerra, o que fez emergir o interesse científico sobre a qualidade de vida (Ribeiro, 2005). Em 1976 Angus Campbell, considerou a qualidade de vida como sendo "uma vaga e etérea entidade, algo sobre o qual muita gente fala, contudo ninguém sabe claramente o que é". Com esta declaração o psicólogo quis mostrar o quão difícil poderia ser a conceitualização deste termo, referindo que este era vago, subjetivo e até poético (Barofsky, 2011).

Durante os anos 80 o conceito de qualidade de vida foi sofrendo alterações devido a vários estudos que foram sendo realizados para uma melhor compreensão sobre a temática (Seidl, \& Zannon, 2004). Na literatura especializada, as definições de qualidade de vida apresentam-se de forma global, enfatizando a satisfação com a vida, dividida em componentes independentes, e que juntos indicariam uma aproximação do conceito geral (Pereira, Teixeira \& Santos, 2012).

Segundo Sampaio (2007), após o período da segunda guerra mundial, a qualidade de vida resumia-se à quantidade de bens materias que as pessoas possuíam. $10 \mathrm{Na}$ perspetiva biológica, o conceito de qualidade de vida baseia-se no conhecimento que a pessoa da sua condição física e das capacidades para realizar as atividades de vida diária. Do ponto de vista cultural, o conceito individual de qualidade de vida é determinado por vários fatores e a sua perceção depende das demandas da sociedade, o desenvolvimento científico e tecnológico e 0 poder económico. Já numa perspetiva económica, a qualidade de vida está relacionada com o valor que as pessoas atribuem aos bens matérias e aos seus valores pessoais. Numa perspetiva psicológica, depende da perspetiva individual e está relacionada com a imagem corporal, a vida profissional, as relações interpessoais, a saúde, a motricidade e a capacidade de realizar as AVD s (Lopes,2013).

A Organização Mundial da Saúde define a qualidade de vida como "a perceção do individuo, da sua posição na vida, no contexto da sua cultura e dos seus valores no qual vive e em relação aos seus objetivos, expetativas, padrões e preocupações" (WHOQOL Group, 1997).

A grande maioria das definições de qualidade de vida relacionada a saúde engloba aspetos do conceito geral de qualidade de vida e dimensões mais específicas relacionadas com a saúde (Phillips, 2006).

Na literatura internacional podemos encontrar vários pontos de vista e diversas definições propostas para 0 conceito de qualidade de vida relacionada a saúde. Para Simonson (1996), ambos os termos, saúde e qualidade 


\section{AVALIAÇÃO DA QUALIDADE DE VIDA NA POPULAÇÃO PORTUGUESA}

de vida, englobam três dimensões, a física, a psicológica e a social, que apesar de distintas estão intimamente relacionadas e são influenciadas pelas experiências individuais, crenças, expetativas e perceções que cada individuo tem da realidade que 0 rodeia.

Ribeiro (1994) refere a existência de dois conceitos de qualidade de vida: o conceito geral, que refere que a qualidade de vida que está dependente das doenças que as pessoas têm, e da análise do impacto destas doenças e do tratamento na qualidade de vida das pessoas; e o conceito específico que se refere às limitações ou à forma como essa doença específica impacta a qualidade de vida.

Segundo Ferrans (2005), a qualidade de vida relacionada a saúde envolve aspetos subjetivos, ligados diretamente aos problemas de saúde, permitindo avaliar o impacto de uma determinada doença na qualidade de vida em geral. 0 autor ainda defende que 0 termo é vulgarmente utilizado para indicar estados de saúde, sintomas, capacidade física, bem-estar, satisfação pessoal e social e felicidade.

No seio das ciências biológicas e humanas tem-se notado uma crescente preocupação com questões relacionadas a saúde e qualidade de vida, no sentido de valorizar parâmetros mais amplos e abrangentes que 0 controle sintomático, aumento da expetativa de vida e diminuição de mortalidade (Fleck, Louzada, Xavier, Chachamovic, Vieira, Santos \& Pinzon).

Naughton e Shumaker (2003) consideraram fundamental a incorporação na avaliação da qualidade de vida relacionada com a saúde de dimensões primárias como a funcionalidade física, psicológica e social, o bem-estar ou a satisfação com vida e a perceção do estado de saúde. Para os especialistas, estas são as dimensões mínimas a serem incluídas para a avaliação e medição da qualidade de vida relacionada com a saúde de forma eficaz e abrangente.

A literatura dá-nos a conhecer alguns fatores diretamente ligados à qualidade de vida relacionada com a saúde. Minayo, Hartz e Buss (2000) citam fatores como as deteriorações funcionais, perceções e condições sociais induzidas pela doença. Já Naughton e Shumaker (2003), valorizam fatores como, o conforto ou sensação de bem-estar, a capacidade de manter atividades a nível físico, mental e intelectual, e atividades relevantes no seio familiar, trabalho e comunidade. Ferrans (2005) dá destaque à fatores como o rendimento, a liberdade e o meio ambiente.

0 conceito de qualidade de vida é complexo e até ambíguo, e existe uma grande variedade de pontos de vista, conceitos e instrumentos de avaliação. Torna-se necessário a realização de estudos no sentido de medir e avaliar a qualidade de vida para dar resposta as necessidades de saúde dos indivíduos.

Torna-se, portanto, indispensável numa ótica de intervenção a necessidade da avaliação dos níveis de qualidade de vida relacionada com a saúde e dos determinantes da mesma.

\section{OBJETIVO}

Assim pretendemos avaliar a qualidade de vida nos indivíduos com idade igual ou superior a 18 anos.

\section{METODOLOGIA}

Para tal, optámos por realizar um estudo descritivo de cariz transversal com uma abordagem quantitativa. Foi usada uma metodologia denominada bola de neve ou snowball sampling, sendo uma técnica de amostragem não probabilística. Foi utilizado um questionário constituído por questões de caraterização e pelo SF-36v2 (The Medical Outcomes Study 36-item Short Form Health Survey). Este é um questionário genérico de saúde, com 36 itens que avaliam a dimensão física, emocional e social, assim como os desempenhos físico e emocional, a dor e a saúde geral e vitalidade. 0 seu conteúdo, tanto referente a saúde física como mental, a robustez psicométrica e a relativa simplicidade são fatores que facilitam a sua utilização. Estes factores, associados à existência de um projecto internacional de adaptação do questionário original em diversos países (International Quality of Life Assessment - IQOLA — Project) estão a transformar o SF-36 Health Survey num dos instrumentos genéricos de medição de estado de saúde com maior potencial na utilização internacional e na avaliação de resultados clínicos (Ferreira, 2000). 
A colheita de dados foie efetuada em 2020. Foram tidos em conta os preceitos éticos aplicados a este tipo de estudos, tendo em conta a Declaração de Helsínquia e Convenção de Oviedo.

Dos critérios de inclusão fizeram parte ter idade igual ou superior a 18 anos, estar consciente e orientado e aceitar, voluntariamente, fazer parte do estudo em questão. Para este efeito, aquando do preenchimento do questionário, as pessoas foram informadas do objetivo do mesmo, assegurando a natureza confidencial, 0 anonimato total dos participantes e que os dados recolhidos seriam utilizados unicamente para fins académicos e de investigação. Ao aceitar prosseguir no questionário, estavam a afirmar que aceitavam participar por livre vontade no estudo, isto é, dar o consentimento informado. 0 consentimento é considerado legal quando obtido de forma livre esclarecida. Como estratégia foi utilizado o Google Forms.

A amostra é assim constituída por 135 pessoas, as quais obedecem aos critérios de inclusão e acederam ao questionário no primeiro trimestre de 2021.

\section{RESULTADOS}

A amostra é constituída por 135 indivíduos, maioritariamente do sexo feminino (68,15\%), com idades compreendidas entre os 18 e os 63 anos. São maioritariamente solteiros (60,74\%), licenciados (50,37\%), residentes em zona urbana $(70,37 \%)$.

Quadro 1 - Caracterização da mostra.

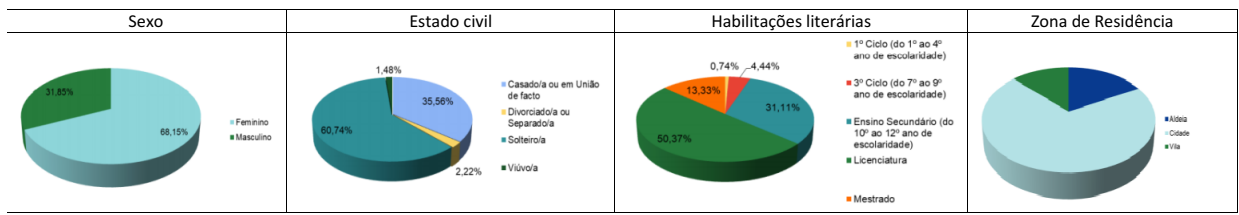

Relativamente ao estado de saúde (Quadro 2), percecionam-na boa e excelente (48,88\%), comparativamente a um ano atrás 45,93\% percecionam-na semelhante. Cerca de metade dos inquiridos não sente limitações relacionadas com a dor $(50,74)$. Em termos físicos, sentem quase sempre vitalidade $37,5 \%$ dos questionados e algumas vezes $27,94 \%$. Em termos psicológicos e emocionais $43,38 \%$ sentem tranquilidade e calma a maior parte do tempo e 23,53 sentem algum tempo. Mais de 50\% não sentem qualquer limitação nas atividades quer física quer psicológica ou emocional. Sentem-se tão saudáveis ou mais como os outros 62,24\%. 


\section{AVALIAÇÃO DA QUALIDADE DE VIDA NA POPULAÇÃO PORTUGUESA}

Quadro 2 - Caracterização do estado de saúde: SF-36

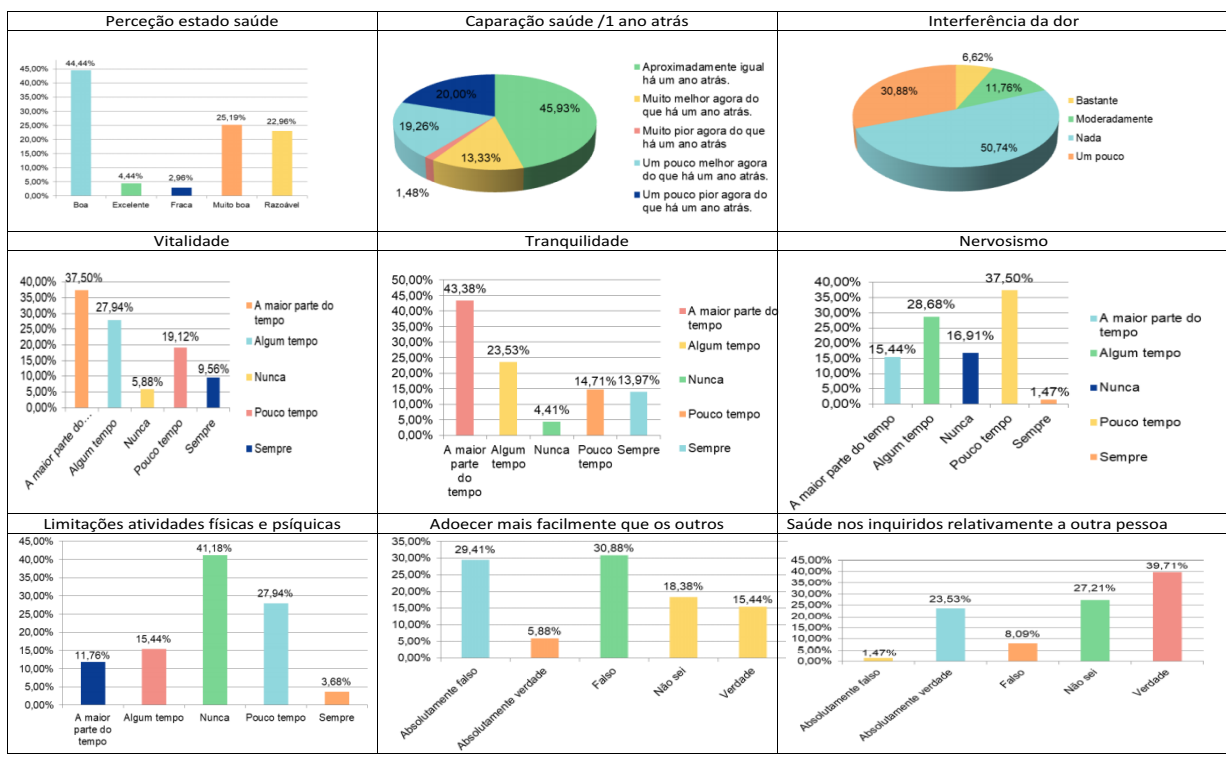

\section{DISCUSSÃO}

Há uma grande falta de consenso conceitual, devido a complexidade do conceito e a utilização por diversas áreas de estudo do termo qualidade de vida. (Farquhar, 1995).

0 termo é amplamente utilizado e apesar de deter definições por vezes controversas, trata-se de uma construção multidimensional, ou seja, a qualidade de vida resulta de várias dimensões inter-relacionadas e não de um conceito unitário. (Silva, Pais-Ribeiro, Cardoso, \& Ramos, 2003)

Segundo a OMS a qualidade de vida é a perceção do individuo, da sua posição na vida, no contexto da sua cultura e dos seus valores no qual vive e em relação aos seus objetivos, expetativas, padrões e preocupações." (WHOQOL Group, 1997).

Tem-se notado uma crescente preocupação no sentido de conhecer e medir o nível de qualidade de vida em saúde. A avaliação deste parâmetro tem-se tornado fundamental para melhorar o estado de saúde das populações e 0 acesso aos cuidados de saúde. 0 incremento dos instrumentos de medida e avaliação da qualidade de vida em saúde na investigação têm permitido conhecer o estado de saúde, comparar populações, deter desigualdades e compreender as causas das assimetrias em saúde. (Lopes, 2013).

Um estudo feito em 2013, baseado nos dados do $4^{0}$ Inquérito Nacional de Saúde, permitiu conhecer os determinantes da qualidade de vida em saúde em Portugal, e concluiu-se que em Portugal o índice de qualidade de vida em saúde varia de acordo com a localização geográfica da amostra, o género, 0 estado civil, o facto de ter ou não um seguro de saúde, as horas semanais de trabalho, entre outros fatores (Lopes, 2013).

\section{CONCLUSÕES}

Estes resultados permitem-nos concluir que em geral os participantes apresentam bons índices de qualidade de vida relacionada. Resultados semelhantes foram encontrados no que respeita à saúde física e saúde emocional (Lopes 2013).

Tendo em conta que atravessamos uma situação pandémica, seria interessante repetir o estudo aquando do fim da situação pandémica para verificar possíveis alterações, principalmente no que se refere à saúde mental da população. 
0 tamanho da amostra pode ser considerado como limite desta investigação. Estas avaliações permitem a identificação dos determinantes da saúde e facilitam aos a tomada de decisão.

\section{REFERÊNCIAS BIBLIOGRÁFICAS}

Barofsky, I. (21 de Abril de 2012). Can quality or quality-of-life be defined? Quality of life research, pp. 625-631.

Farquhar, M. (1995). Definitions of quality of life: a taxonomy. Journal of Advanced Nursing, 502-508.

Ferrans, C. E. (2005). Definitions and conceptual models of quality of life. . Cambridge: cambridge university

Ferreira, P. (2000). Criação da Versão Portuguesa do MOS SF-36, Parte I - Adaptação Cultural e Linguística. Revista Acta Médica Portuguesa, 55-66.60

Fleck, M., Leal, O., Louzada, S., Xavier, M., Chachamovich, E., Vieira, G., Santos, L., Pinzon, V. (1999). Desenvolvimento da versão em português do instrumento de avaliação de qualidade de vida da OMS (WHOQ0L-100). Rer Bras Psiquiatr, 21 (1)

Fleck, M. P. (2000). 0 instrumento de avaliação de qualidade de vida da Organização Mundial de Saúde (WHOQ0L-100) . Ciência e Saúde Colectiva, pp. 33-38.

Fleck, M. P. (2000). Aplicação da versão em português do instrumento abreviado deavaliação da qualidade de vida "WHOQOL-bref". Revista de Saúde Pública, 178-183.

Guyatt, G. F. (1993). Measuring health-related quality of life. Annals of Internal Medicine. pp. 622-629.

Melo, P. (2020). Enfermagem de Saúde Comunitária e de Saúde Pública (1ª ed.). Lisboa: Lidel.

Minayo, M.C.S.; Hartz, Z.M.A.; Buss, P.M. Qualidade de vida e saúde: um debate necessário. Ciência \& Saúde Coletiva, Rio de Janeiro, v.5, n.1, p.7-18, 2000

Monahan, S. N. (2009). Phips Enfermagem Médico-Cirúrgica. Lusodidacta.61

Nunes, R. \&. Rego (2002). Prioridades na Saúde ( $2^{\circledR}$ ed.). Lisboa: Editora McGraw-hill.

OE. (2011). Padrões de Qualidade dos Cuidados de Enfermagem: Enquadramento conceptual; Enunciados descritivos. 1-11. Lisboa: Ordem dos Enfermeiros.

Pereira, Teixeira \& Santos (2012). Qualidade de vida: abordagens, conceitos e avaliação. Rev. bras. Educ. Fís. Esporte, São Paulo, v.26, n.2, p.241-50

Ribeiro, J. L. (1994). Análise Psicológica. A importância da qualidade de vida para a Psicologia da Saúde, pp. 79-191.

Ribeiro, J. L. (2005). Introdução à Psicologia da Saúde. Coimbra: Editora Quarteto. Ribeiro, J. L. (2009). A importância da qualidade de vida para a Psicologia da Saúde. Alcochete: Textiverso.

Sampaio, A, C, L. (2007). Benefícios da caminhada na qualidade de vida dos adultos. Dissertação de licenciatura apresentada na Faculdade de Desporto da Universidade do Porto.

Seidl, E. M. (2004). Cadernos de Saúde Pública. Cadernos de Saúde Púbica, 580- 588.

Sieber, W. J. (2008). Quality of well being - Self-Administered (QWB-SA) Scale, User s Manual. San Diego: University of California.

Silva, I. P.-R. (21 de 2 de 2003). Qualidade de vida e complicações crónicas da diabetes. Análise Psicológica,, pp. 185-194.

Testa MA, Simonson DC. Assessment of quality of life outcomes. New Eng J Med 1996;334:835-40

Turato, E. (2003). Tratado da metodologia da pesquisa clínico-qualitativa: construção teórico-epistemológica, discussão comparada e aplicação nas áreas da saúde e humanas. Petrópolis: Vozes 
